\title{
Proceeding
}

Supplementary Issue: Autumn Conferences of Sports Science. Costa Blanca Sports Science Events, 18-19 December 2020. Alicante, Spain.

\section{Linking psycho-physical profiles to different training condition during COVID-19 confinement}

\author{
VALERIO BONAVOLONTÀ, STEFANIA CATALDI $\triangle$, ANTONELLO CONENNA, FRANCESCO FISCHETTI \\ School of Medicine, Department of Basic Medical Sciences, Neuroscience and Sense Organs, University of \\ Study of Bari "Aldo Moro", Bari, Italy
}

\begin{abstract}
At the end of the period of home confinement due to Covid-19 lockdown, the 12-item Health Survey (SF-12) questionnaire was used to assess the quality of life. Participants also completed the Profile of Mood State (POMS) for the assessment of mood. Data were collected through an online form on a sample of 143 adults (71\% aged $18-30,12 \% 31-40,11 \% 41-50$ and $6 \%$ aged $>50,58 \%$ males and $42 \%$ females). Subjects were divided into 5 groups according to the corresponding training condition followed during the first lockdown. Training classes were carried out via online guided distance learning. 19 subjects (Group FT) received functional training classes as they did before the confinement. Group FTL (29 subjects) followed another functional training only during the lockdown. Group DT (46 subjects) continued a non-functional type training also during lockdown; group TL performed one training only during lockdown. Finally, group NT (25 subjects) received no training. Results from SF-12 showed no significant differences between groups. POMS result indicated that only the FT group had positive effects on anxiety and depression, whereas a functional training performed with a different program (FTL) resulted in no significant effects on anxiety compared to NA group. Keywords: Functional training; Anxiety; Depression; Quality of life; Home training; Distance learning.

Cite this article as:

Bonavolontà, V., Cataldi, S., Conenna, A., \& Fischetti, F. (2021). Linking psycho-physical profiles to different training condition during COVID-19 confinement. Journal of Human Sport and Exercise, 16(2proc), S697-S704. doi:https://doi.org/10.14198/ihse.2021.16.Proc2.55
\end{abstract}

Corresponding author. School of Medicine: Department of Basic Medical Sciences, Neuroscience and Sense Organs. University of Study of Bari "Aldo Moro", Lungomare Starita 1/b, 70132, Bari. Italy. https://orcid.org/0000-0002-5929-4766 E-mail: stefania.cataldi@uniba.it Abstract submitted to: Autumn Conferences of Sports Science. Costa Blanca Sports Science Events, 18-19 December 2020. Alicante, Spain.

JOURNAL OF HUMAN SPORT \& EXERCISE ISSN 1988-5202

(C) Faculty of Education. University of Alicante

doi:10.14198/jhse.2021.16.Proc2.55 


\section{INTRODUCTION}

Many studies that support the positive effects of physical activity on mental health and in particular with regard to anxiety, depression and quality of life, and it is clear that physical activity can lead to many physiological changes that result in improved mood, self-esteem, stress and anxiety levels (Abd El-Kader and Al-Jiffri, 2016; Anderson and Shivakumar, 2013; Bartholomew et al., 2005; Broman-Fulks et al., 2004; Chang et al.,2017; DeBoer et al., 2012).

However, currently in the literature it is not clear which physical activity is better in order to produce an improvement of these parameters, as improvements can be achieved either with a purely aerobic and with a purely anaerobic exercise (DiLorenzo et al.,1999; Martinsen et al.,1989).

In addition, there are numerous studies in the scientific literature reporting that exercise improves mood disorders, primarily depression (Kvam et al.,2016) and anxiety (Wipfli et al., 2008) in clinical and nonclinical populations; in fact, exercise as a nonpharmacological treatment has been shown to be superior to control interventions and sometimes comparable to medication for mild and moderate depression (Wipfli et al., 2008; Cooney et al., 2013).

Major depressive disorder (MDD) is one of the major diseases worldwide that affects health status more than diseases such as diabetes and diseases to the musculoskeletal system, thus it should be considered a public health priority (Moussavi et al., 2007).

In fact, depression causes a marked change in mood such as sadness and irritability and is accompanied by numerous psychophysiological alterations including appetite and sleep disturbance, loss of ability to feel pleasure, slowed speech and action, and suicidal thoughts (Belmaker and Agam; 2008).

Therefore, physical activity and in particular physical exercise, are to be considered an important antidepressant therapy option based on various evidence (Schuch et al., 2016, Vancampfort et al., 2017), and has been included in World Health Organization (WHO) guidelines as a standard treatment of depression (WHO, 2012).

Functional training (FT) is often performed using an interval training methodology, where interval training is defined as "a training in which we alternate periods of relatively intense exercise with periods of lower intensity effort or complete rest for recovery" (Gibala et al., 2014). The two most commonly used forms of interval training are sprint interval training (SIT) and high intensity interval training (HIIT) (Gibala et al., 2014).

SIT refers to protocols that are performed at supramaximal exercise at intensities corresponding to loads/intensities greater than those associated with maximum oxygen consumption $\left(\mathrm{VO}_{2 \max }\right)$ obtained with an incremental test (Viana et al., 2018); whereas HIIT is a training stimulus with a target intensity between $80 \%$ and $100 \%$ of maximum heart rate (FC max) as reported by Viana et al. (2018).

From a study conducted by Lee et al. (2014) it is showed that exercise can increase neurotrophin levels and, in particular, HIIT has been shown to improve depression and neuronal plasticity in the hippocampus in rats with post-stroke depression (Luo et al., 2019); these studies hypothesized that both HIIT and SIT would improve depressive and anxiety symptoms in healthy women. Another study conducted by Wu et al. (2015) showed the success of HIIT training with psychiatric patients and in addition a study conducted by Heggelund et al. (2014) demonstrated the positive effect of this type of training on subjects who suffered from depression. 
In addition, another study (Kvam et al., 2016) have demonstrated the positive effects of SIT training on subjects suffering from depression. Moreover, the WHO places specific recommendations for home physical training, recommending 60' per day of moderate to vigorous physical activity for children and youth aged 6 17 years and $75^{\prime}$ per week of vigorous physical activity or 150' per week of moderate physical activity for adults and the elderly; all of which can help counteract the harmful physical and mental side effects of lifestyle regulations due to Covid-19 (Hammami et al., 2020).

Therefore, the aim of the study is to verify if physical activity and, specifically, a functional training program can have positive effects on anxiety, depression, and quality of life in healthy subjects during the lockdown period due to COVID-19, compared to subjects who did not engage in any type of physical activity during the same period.

\section{METHODS}

\section{Participants}

The sample consisted of 143 participants (71\% aged 18-30, 12\% 31-40,11\% 41-50 and 6\% aged >50; 83 females and 60 males) who decided to voluntarily complete a questionnaire created through Google Forms. This questionnaire was submitted in the week immediately after the end of the severe lockdown had in Italy due to the COVID-19 pandemic that began on March 9, 2020 and ended on May 18, 2020. The inclusion criteria for the present study were to be healthy and at least 18 years of age.

The sample was divided into 5 groups: the functional training group (FT) composed of 19 people who performed in the lockdown period the training protocol of 10 weeks through video lessons. This group performed functional training during the lockdown period but was also performing the same type of training before the lockdown. A second group of 29 people performed functional training only during this lockdown period (FTL) in a self-taught manner or following a different training protocol; before the lockdown they performed another type of training. Another group of 46 people performed a different type of training (DT) than functional training both during the lockdown and before. A fourth group of 24 people trained during the lockdown period (TL) but were not performing any type of physical activity before the lockdown. Finally, a group of 25 people did not perform any type of training during the lockdown period (NT) nor did before the lockdown.

\section{Instruments}

The questionnaire used for data collection consisted of three sections: the first in which biographical information were provided, which type of physical activity was performed or not performed before and during the lockdown, the frequency of activity in terms of days and hours of training per week, and finally whether or not any training was planned by a specialist in sport science or performed autonomously.

In the second section there was a validated questionnaire as the SF12 (Ware, 1996) which aims to assess the health status of the subject in particular what the subject thinks of his health. This questionnaire consists of 12 questions rated from 1 to 6 for some items, from 1 to 5 for others and from 1 to 2 for the resting, with a total score that can vary from a minimum total score of 12 and a maximum of 47 . SF12 questionnaire allows to calculate two indices, one of which concerns the physical status of the group, referred to as Physical Component Summary (PCS): this index takes into account six items in turn referred to various areas; in particular for physical activity, physical health, physical pain and to health in general. The other index analysed is the Mental Component Summary (MCS) which is an index that measures the mental state of the 
group: even for this index six items are referred to various areas, in particular to vitality, social activity, emotional state and to mental health.

In the third section the participant had to answer a standardized and validated questionnaire such as the abbreviated Profile of Mood State (POMS-40; Grove and Prapavessis,1992), composed of 40 questions rated from 0 to 4; by answering the items of this questionnaire indications on the scales of tension, anger, fatigue, depression, affection related to esteem, vigour and confusion were provided. Total mood disorder (TMD) is calculated which is a value that includes seven subscales (tension, anger, fatigue, depression, vigour, confusion and effect).

\section{Procedures}

The training protocol used to carry out functional training performed with interval training methodology included 8 different exercises for each training session, performed alternating a phase of work and a phase of passive recovery with a work/recovery ratio of 1:1; each week included two training sessions with an average duration of $1 \mathrm{~h}$ for each lesson. Each training session or workout was divided into three parts: a part of activation or warm up in which exercises of joint mobility and dynamic stretching were performed with an average duration of $10^{\prime}$. A central phase lasting about 32 ' with a workout consisting of eight exercises performed with an interval training methodology that alternated a work phase of 30 " and a passive recovery phase of 30". Finally, a final cool down phase lasting about 10' in which static stretching exercises were performed. For all the training conditions the average training frequency was twice a week for about one hour.

\section{Statistical analysis}

This study has a cross-sectional design. Considering a $95 \%$ confidence level or $a=.05$, using the ShapiroWilk test it was seen that the distribution for the variables PCS, MCS and total score for the SF-12 (TOT SF12) was not normal. Given the non-normal distribution the non-parametric Kruskall-Wallis test was used to check for statistical differences between the five groups for each dependent variable. When a statistical significance between groups for any dependent variable was detected, a two-tailed Mann-Whitney test was applied to verify which groups showed the differences. Statistical significance was set at $p<.05$.

\section{RESULTS}

Data analysis showed that there were no statistically significant differences between the five groups regarding the health status collected with the questionnaire SF-12, as the TOT SF-12 value of the five groups was $p=$ .334; also, analysing in detail the two indices of the questionnaire, PCS and MCS, no statistically significant difference was found because the $p$-value was .359 for PCS and $p=.36978$ for MCS index.

Conversely, with regard to the POMS 40 , statistically significant differences were found between the five groups. In particular, for the variable indicating the POMS 40 total mood disorder score (TMD) these differences were highlighted comparing the FT and NT groups $(p=.01878)$, the DT and NT groups $(p<.001)$, between the DT and FTL $(p=.01208)$ groups and finally between the DT and TL groups $(p=.0151)$.

\section{DISCUSSION AND CONCLUSIONS}

Aim of the study was to compare different training conditions performed before and during the lockdown home-confinement linked to psycho-physical parameters in healthy adults. Reduced level of physical activity can alter bio-psyco-social functioning and wellbeing (D'Elia et al. 2020). Previous research by the authors 
showed that a functional training, such as CrossFit could positively affect the general physical well-being and mental attitude and improve the emotional perceived self-efficacy (Bonavolontà et al., 2020 b). In addition, several studies reported that physical and sport activities were deeply influenced by the current pandemic scenario as online distance learning allowed to attend different classes as well as to maintain a certain physical fitness for sport and individual teams (Bonavolontà et al. 2020; Raiola et al. 2020). Raiola et al. (2020) reported that functional training was practiced by $27 \%$ of a 268 people sample during the first Italian lockdown. In addition, it is crucial to understand which modality better fit into the current limited socioeconomic scenario, to find the better strategy in order to design efficient teaching-learning experiences (Sgrò et al. 2018). Indeed, as reported by Haga et al. (2018) different cultural contexts, lifestyles and physical activity contexts can impact on a successful process of developing motor competences.

Our results showed no significant differences in relation to SF-12 questionnaire for all the training conditions, i.e., between subjects who engaged in physical activity or a functional training program during the lockdown period, and those who did not engage in any type of physical activity.

Conversely, statistically significant differences were found for POMS-40. Indeed, with regard to the variable TMD, comparing the means of the groups, FT was significantly lower than NT group: thus, the group that has performed functional training (FT) following a 10-week training protocol for the entire period of lockdown through video lessons created by a specialist in sport and exercise science, showed a positive effect on the total mood disorder (TMD) compared to the NT group that did not perform any type of training during the lockdown nor was doing before the lockdown. From the comparison of the DT and NT groups, it can be seen that DT, that was the group that has performed a different type of training than the functional during the lockdown and before, showed a significantly lower TMD than NT, suggesting a positive effect on total mood disorder. From the comparison of the DT and FTL groups comparing the means of the two groups, DT value is significantly lower than that of FTL which represents the group that has done a functional training in a selftaught manner or following a different training protocol and that before the lockdown had a different type of training than the functional: this implies that the DT had a positive effect on TMD respect to the FTL group. Finally, comparing the DT and TL groups, DT has significantly lower mean than TL that was the group that has trained exclusively during the lockdown, suggesting that the DT had a greater positive impact on TMD than the TL group. On the other hand, with regard to the comparisons between the FT and FTL groups, between the FT and DT groups, between the FT and TL groups, between the NT and FTL groups, between the NT and TL groups, and finally between the FTL and TL groups, no statistically significant differences were found. It should be noted that the same type of functional training performed in a self-taught manner or by performing a different type of training protocol $(F T L)$, did not determine any statistically significant beneficial effect compared to the group that did not perform any type of training (NT). Our findings are partly in line with Viana and colleagues (2019) who found that both HIIT and SIT groups similarly improved depressive symptoms but not anxiety levels in healthy and physically active young adult women.

Although some research highlighted that digital devices could cause and increase mental fatigue (Greco et al., 2017), currently, online distance training allowed to keep a relationship between the teacher/trainer and his athletes (Bonavolontà et al., 2020), like it has occurred for several participants to the present study.

Limitations of the study include the fact that gender has not been considered, while previous research reported that physical education plays a gender-related role on body dissatisfaction (Fischetti et al., 2020) and functional training does as well on anxiety and depression (de Sousa et al., 2020). 
Future extension of the present work should include a longitudinal design, consistent with contingent epidemiologic needs and limitations.

In conclusion, subjects who remained active, in particular those who have continued to perform the same type of training done before the lockdown, had a better profile from the point of view of anxiety and depression compared to those who have not done any type of training, to those who changed their usual training during the lockdown by choice or by necessity performing workouts in a self-taught manner, and also compared to those who began to train only in this period of total lockdown.

\section{REFERENCES}

Abd El-Kader, S. M., \& Al-Jiffri, O. H. (2016). Aerobic exercise improves quality of life, psychological wellbeing and systemic inflammation in subjects with Alzheimer's disease. African health sciences, 16(4), 1045-1055. https://doi.org/10.4314/ahs.v16i4.22

Anderson, E. H., \& Shivakumar, G. (2013). Effects of exercise and physical activity on anxiety. Frontiers in psychiatry, 4, 27. https://doi.org/10.3389/fpsyt.2013.00027

Bartholomew, J. B., Morrison, D., \& Ciccolo, J. T. (2005). Effects of acute exercise on mood and wellbeing in patients with major depressive disorder. Medicine and science in sports and exercise, 37(12), 2032. https://doi.org/10.1249/01.mss.0000178101.78322.dd

Belmaker, R. H., \& Agam, G. (2008). Major depressive disorder. New England Journal of Medicine, 358(1), 55-68. https://doi.org/10.1056/NEJMra073096

Bonavolontà, V., Cataldi, S., Maci, D., \& Fischetti, F. (2020). Physical activities and enjoyment during the lockdown: Effect of home-based supervised training among children and adolescents. Journal of Human Sport and Exercise, 15 (Proc. 4), S1338 - S1343. https://doi.org/10.14198/ihse.2020.15.Proc4.31

Bonavolontà, V., Greco, G., Cataldi, S., De Florio, O., \& Fischetti, F. (2020). Effects Of An 8-week Crossfit Program On Psychophysical Well-being In Healthy Adolescents: Medicine \& Science in Sports \& Exercise, 52(7S), 279. https://doi.org/10.1249/01.mss.0000676608.61263.8e

Broman-Fulks, J. J., Berman, M. E., Rabian, B. A., \& Webster, M. J. (2004). Effects of aerobic exercise on anxiety sensitivity. Behaviour research and therapy, 42(2), 125-136. https://doi.org/10.1016/S0005-7967(03)00103-7

Chang, Y. C., Lu, M. C., Hu, I. H., Wu, W. C. I., \& Hu, S. C. (2017). Effects of different amounts of exercise on preventing depressive symptoms in community-dwelling older adults: a prospective cohort study in Taiwan. BMJ open, 7(4), e014256. https://doi.org/10.1136/bmjopen-2016-014256

Cooney, G. M., Dwan, K., Greig, C. A., Lawlor, D. A., Rimer, J., Waugh, F. R., \& Mead,G. E. (2013). Exercise for depression. Cochrane database of systematic reviews, (9). https://doi.org/10.1002/14651858.CD004366.pub6

DeBoer, L. B., Powers, M. B., Utschig, A. C., Otto, M. W., \& Smits, J. A. (2012). Exploring exercise as an avenue for the treatment of anxiety disorders. Expert review of neurotherapeutics, 12(8), 1011 1022. https://doi.org/10.1586/ern.12.73

D'Elia, F., Tortella, P., Sannicandro, I., \& D'Isanto, T. (2020). Design and teaching of physical education for children and youth. Journal of Human Sport and Exercise, 15(4proc), S1527-S1533. https://doi.org/10.14198/ihse.2020.15.Proc4.48

de Sousa Junior, I., Nunes, R. S. M., de Luca Corrêa, H., \& Vieira, E. (2020). Functional training program: the impact on depression, anxiety and sleep quality in adolescents. Sport Sciences for Health, 1-10. https://doi.org/10.1007/s11332-020-00679-7 
DiLorenzo, T. M., Bargman, E. P., Stucky-Ropp, R., Brassington, G. S., Frensch, P. A., \& LaFontaine, T. (1999). Long-term effects of aerobic exercise on psychological outcomes. Preventive medicine, 28(1), 75-85. https://doi.org/10.1006/pmed.1998.0385

Fischetti, F., Latino, F., Cataldi, S., Greco, G. (2020) Gender differences in body image dissatisfaction: The role of physical education and sport. Journal of Human Sport and Exercise, 15 (2), pp. 241-250. https://doi.org/10.14198/hhse.2020.152.01

Gibala, M. J., Gillen, J. B., \& Percival, M. E. (2014). Physiological and health-related adaptations to lowvolume interval training: influences of nutrition and sex. Sports Medicine, 44(2), 127-137. https://doi.org/10.1007/s40279-014-0259-6

Greco, G., Tambolini, R., Ambruosi, P., Fischetti, F. (2017) Negative effects of smartphone use on physical and technical performance of young footballers, Journal of Physical Education and Sport, 17 (4), art. no. 280, pp. 2495-2501.

Grove, J. R., \& Prapavessis, H. (1992). Preliminary evidence for the reliability and validity of an abbreviated Profile of Mood States. International Journal of Sport Psychology, 23(2), 93-109.

Haga, M., Tortella, P., Asonitou, K., Charitou, S., Koutsouki, D., Fumagalli, G., \& Sigmundsson, H. (2018). Cross-cultural aspects: Exploring motor competence among 7-to 8-year-old children from Greece, Italy, and Norway. Sage open, 8(2), 2158244018768381. https://doi.org/10.1177/2158244018768381

Hammami, A., Harrabi, B., Mohr, M., \& Krustrup, P. (2020). Physical activity and coronavirus disease 2019 (COVID-19): specific recommendations for home-based physical training. Managing Sport and Leisure, 1-6. https://doi.org/10.1080/23750472.2020.1757494

Heggelund, J., Kleppe, K. D., Morken, G., \& Vedul-Kjelsais, E. (2014). High aerobic intensity training and psychological states in patients with depression or schizophrenia. Frontiers in psychiatry, 5, 148. https://doi.org/10.3389/fpsyt.2014.00148

Lee, I. T., Fu, C. P., Lee, W. J., Liang, K. W., Lin, S. Y., Wan, C. J., \& Sheu, W. H. H. (2014). Brainderived neurotrophic factor, but not body weight, correlated with a reduction in depression scale scores in men with metabolic syndrome: a prospective weight- reduction study. Diabetology \& Metabolic Syndrome, 6(1), 18. https://doi.org/10.1186/1758-5996-6-18

Luo, L., Li, C., Deng, Y., Wang, Y., Meng, P., \& Wang, Q. (2019). High-intensity interval training on neuroplasticity, balance between brain-derived neurotrophic factor and precursor brain-derived neurotrophic factor in poststroke depression rats. Journal of Stroke and Cerebrovascular Diseases, 28(3), 672-682. https://doi.org/10.1016/i.jstrokecerebrovasdis.2018.11.009

Martinsen, E. W., Hoffart, A., \& Solberg, Ø. Y. (1989). Aerobic and non-aerobic forms of exercise in the treatment of anxiety disorders. Stress Medicine, 5(2), 115-120. https://doi.org/10.1002/smi.2460050209

Moussavi, S., Chatterji, S., Verdes, E., Tandon, A., Patel, V., \& Ustun, B. (2007). Depression, chronic diseases, and decrements in health: results from the World Health Surveys. The Lancet, 370(9590), 851-858. https://doi.org/10.1016/S0140-6736(07)61415-9

Raiola, G., Aliberti, S., Esposito, G., Altavilla, G., D'Isanto, T., \& D'Elia, F. (2020). How has the Practice of Physical Activity Changed During the COVID-19 Quarantine? A Preliminary Survey. Teoriâ ta Metodika Fizičnogo Vihovannâ, 20(4), 242-247. https://doi.org/10.17309/tmfv.2020.4.07

Schuch, F. B., Vancampfort, D., Richards, J., Rosenbaum, S., Ward, P. B., \& Stubbs, B. (2016). Exercise as a treatment for depression: a meta-analysis adjusting for publication bias. Journal of psychiatric research, 77, 42-51. https://doi.org/10.1016/i.jpsychires.2016.02.023

Sgrò, F., Pignato, S., \& Lipoma, M. (2018). Assessing the impact of gender and sport practice on students' performance required in team games. Journal of Physical Education and Sport, 18, 497 502. 
Vancampfort, D., De Hert, M., Knapen, J., Wampers, M., Demunter, H., Deckx, S., .. \& Probst, M. (2011). State anxiety, psychological stress and positive well-being responses to yoga and aerobic exercise in people with schizophrenia: a pilot study. Disability and rehabilitation, 33(8), 684-689. https://doi.org/10.3109/09638288.2010.509458

Viana, R. B., de Lira, C. A. B., Naves, J. P. A., Coswig, V. S., Del Vecchio, F. B., Ramirez-Campillo, R., ... \& Gentil, P. (2018). Can we draw general conclusions from interval training studies? Sports Medicine, 48(9), 2001-2009. https://doi.org/10.1007/s40279-018-0925-1

Viana, R. B., Gentil, P., Naves, J. P. A., Rebelo, A. C. S., Santos, D. A. T., Braga, M. A. O., \& de Lira, C. A. B. (2019). Interval Training Improves Depressive Symptoms But Not Anxious Symptoms in Healthy Women. Frontiers in psychiatry, 10. https://doi.org/10.3389/fpsyt.2019.00661

Ware, J. E. (1996). SF-12: an even shorter health survey. Med Outcome Trust Bull, 4, 2.

Wipfli, B. M., Rethorst, C. D., \& Landers, D. M. (2008). The anxiolytic effects of exercise: a meta-analysis of randomized trials and dose-response analysis. Journal of Sport and Exercise Psychology, 30(4), 392-410. https://doi.org/10.1123/jsep.30.4.392

World Health Organization. (2012). Depression (N 369) [Fact sheet].

Wu, M. H., Lee, C. P., Hsu, S. C., Chang, C. M., \& Chen, C. Y. (2015). Effectiveness of high-intensity interval training on the mental and physical health of people with chronic schizophrenia. Neuropsychiatric disease and treatment, 11, 1255. https://doi.org/10.2147/NDT.S81482

\section{@(@) $\Theta \Theta$}

This work is licensed under a Attribution-NonCommercial-NoDerivatives 4.0 International (CC BY-NC-ND 4.0). 